\title{
Hipodontia de dentes permanentes: prevalência e distribuição numa População Brasileira
}

\section{Permanent teeth Hypodontia: prevalence and distribution in a Brazilian Population}

\author{
1 Sérgio Elias Vieira Cury sergiocury@usp.br \\ 2 Jessica Ribas de Paula \\ 2 Priscilla Alves dos Santos \\ 1 Maria Dorotéa Pires Neves Cury
}

1 Docente do curso de Odontologia do Centro Universitário de Volta Redonda, UniFOA.
2 Discente do curso de Odontologia do Centro Universitário de Volta Redonda, UniFOA.

\section{RESUMO}

O termo Hipodontia tem sido usado para descrever agenesias de um a 6 dentes, excluídos os terceiros molares Objetivo: avaliar a prevalência e a distribuição de hipodontia numa população, através de análise de radiografias panorâmicas. Metodologia: Cinco mil quinhentas e oitenta radiografias panorâmicas pertencentes ao arquivo da Disciplina de Patologia Bucal do Curso de Odontologia do UniFOA, Volta Redonda, Rio de Janeiro, obtidas no período de janeiro a dezembro de 2014 , foram avaliadas sobre a presença de imagens compatíveis com hipodontia. Resultados: Dentre as 5.580 radiografias examinadas, foram encontrados $77(1,38 \%)$ de indivíduos com imagem correspondente à ausência do germe dentário. A idade variou de 6 a 54 anos, com média de 17,5 anos. Ocorreram 40 casos no gênero feminino $(51,95 \%)$ e 37 no masculino $(48,05 \%)$, numa relação $1,08 \times 1$. $02^{\circ}$ pré-molar inferior foi o dente mais afetado, com $57,60 \%(n=72)$, seguido do pré-molar superior com 22,40\% $(n=28)$. Conclusão: A prevalência de indivíduos com imagem correspondente à ausência do germe dentário foi de $1,38 \%$, sendo o gênero feminino ligeiramente mais acometido pela alteração. 0 dente mais frequentemente envolvido foi o $2^{\circ}$ prémolar inferior, seguido do $2^{\circ}$ pré-molar superior.

\section{PALAVRAS-CHAVE}

Agenesia dentária, Hipodontia, Anomalia dentária, Patologia Bucal.

\begin{abstract}
The term Hypodontia has been used to describe agenesis from one to six teeth, excluding third molars. Objective: To evaluate the prevalence and distribution of hypodontia in a population through analysis of panoramic radiographs. Methodology: Five thousand five hundred and eighty panoramic radiographs belonging to the Oral Pathology of the Dental School of UniFOA, Volta Redonda, Rio de Janeiro, obtained from January to December 2014, with the presence of compatible hypodontia images were evaluated. Results: Among the 5,580 radiographs examined, 77 (1.38\%) were found with image corresponding to the absence of the dental germ. The age range varied from 6 to 54 years old, with the average of 17.5 years old. There were 40 cases in female gender $(51.95 \%)$ and 37 in male gender (48.05\%), in a relation $1,08 \times 1$. The second lower premolar was the most affected tooth, with $57.60 \%(n=72)$, followed by the upper premolar with $22.40 \%(n=28)$. Conclusion: The prevalence of individuals with image corresponding to the absence of the dental germ was $1.38 \%$, being the female gender slightly more affected by the modification. The most frequent tooth involved was the second lower premolar followed by the second upper premolar.
\end{abstract}

\section{KEYWORDS}

Tooth agenesis, Hypodontia, Dental Anomaly, Oral Pathology.

\section{Como você deve citar?}

CURY, Sérgio Elias Vieira; PAULA, Jessica Ribas de; SANTOS, Priscilla Alves dos; CURY, Maria Dorotéa Pires Neves. Características do uso de fármacos sem prescrição por graduandos em Enfermagem. Cadernos UniFOA, Volta Redonda, n. 29, p. 137-147, dez. 2015. 


\section{INTRODUÇÃO}

Do ponto de vista da evolução genética, a dentição dos mamíferos é considerada um sistema de órgãos arranjados sequencialmente, em que números específicos de dentes estão distribuídos em localizações definidas ao longo de eixos lineares dos ossos maxilares (NANCl, 2013).

A formação da dentição é um processo fascinante, que engloba uma complexa série de interações epitélio/mesenquimais, envolvendo diversos fatores, não sendo surpresa que este complexo processo esteja sujeito aos distúrbios que possam causar alterações no desenvolvimento dos dentes (KAPÁDIA; MUES; D'SOUZA, 2007).

Agenesia dentária, também definida como ausência congênita é caracterizada pela redução numérica dos elementos dentários. É uma das anomalias dentárias mais frequentes do ser humano e resulta de distúrbio durante os estágios de iniciação e proliferação na formação dentaria. Pode ser classificada como hipodontia, oligotontia ou anodontia (ROBINSON; MILLER, 1990; KIGNEL, 2013; REGEZI; SCIUBBA; JORDAN, 2013). 0 termo Hipodontia é usado para descrever agenesias de um a 6 dentes, excluídos os terceiros molares; Oligodontia, a ausência de mais de seis dentes; e Anodontia, a ausência completa dos dentes (NEVILLE et al., 2009; SAPP; EVERSOLI; WYSOCKI, 2012; REGEZI; SCIUBBA; JORDAN, 2013).

O processo de desenvolvimento dentário está sob estrito controle genético, sugerindo que formas herdadas de agenesias dentárias são causadas pela interrupção de um ou mais processos moleculares que regulam a formação dentária (NANCl, 2013). Essas anomalias têm sua origem na odontogênese, principalmente durante as fases de iniciação e proliferação do germe diretamente da lâmina dentária ao longo do período de desenvolvimento intra-uterino do bebê (BRUNNER; GUEDES PINTO,1983).

A etiologia da agenesia dentária é multifatorial, incluindo, além da predisposição genética, fatores externos, radiação e síndromes (NEVILLE et al., 2009; SAPP; EVERSOLE; WYSOCKI, 2012). Sua prevalência é variada, sendo relatada de 0,03 a $10,1 \%$, com maior incidência no gênero feminino, numa proporção de 1,5X1 em relação ao masculino (FARIAS et al., 2006; SCULLY, 2009; GOKKAYA; MOTRO; GARKUL, 2015).

A hipodontia de dentes posteriores, com exceção dos $3^{\circ}$ molares, pode gerar graves problemas peridontais e oclusais, como oclusão traumática, inclinações indesejáveis, surgimento de diastemas que causam impactação alimentar, com consequentes danos ao periodonto interdental. Por outro lado, a hipodontia de dentes anteriores causa uma estética indesejável e prováveis problemas fonéticos. Para o diagnóstico dessa anomalia são de grande importância os exames radiográficos, juntamente com o exame clinico, para um bom planejamento e terapêutica para cada caso (BORBA et al., 2010).

Tendo suas bases estabelecidas em 1954 por Paatero em Helsink na Finlândia (PAATERO, 1959), a radiografia panorâmica, dada a sua abrangência, logo se tornou instrumento de qualidade inquestionável no delineamento do perfil epidemiológico de diversas condições mórbidas que acometem o terço médio-inferior da face. Reconhece-se a radiografia panorâmica como a melhor imagem convencional para visualização das anomalias maxilo-mandibulares, dentre outras indicações (GIRONDI et al., 2006), sendo a mais indicada para estudo de agenesia dentária por registrar todo o complexo maxilomandibular em uma tomada única, e por possuir menor índice de radiação quando comparada à tomada de radiografias periapicais de todos os dentes (PAULA; FERRER, 2007; GARTNER; GOLDENBERG, 2009).

Diante do exposto, torna-se indispensável o conhecimento da prevalência de hipodontia, contribuindo na compreensão da sua importância clínica, associada aos possíveis problemas provocados no 
sistema estomatognático, dentre eles a má-oclusão, culminando em alterações funcionais e estéticas no indivíduo.

O objetivo desse estudo é avaliar a prevalência e a distribuição de hipodontia numa população, através de análise de radiografias panorâmicas.

\section{REVISÃO DE LITERATURA}

Oliveira et al. (2001) avaliaram 284 alunos do curso de Odontologia da Universidade de Taubaté, em São Paulo, sobre a prevalência de hipodontia. Os resultados mostraram que, dos 284 alunos examinados, $11(3,87 \%)$ apresentaram hipodontia. Os dentes ausentes mais frequentemente encontrados foram os incisivos laterais e pré-molares.

Girondi et al. (2006) estudaram a ocorrência de anomalias dentárias de desenvolvimento numa população com idade variando de 12 a 25 anos, na cidade de Bragança Paulista. No trabalho foram avaliadas 533 radiografias panorâmicas, de pacientes dos sexos feminino e masculino. Os autores relataram uma prevalência de $6,57 \%$ de anodontias, sendo o dente mais envolvido o terceiro molar inferior.

Um estudo com alunos de graduação da faculdade de Odontologia da UNESP de Araçatuba foi realizado em 2006 por Castro et al., no intuito de avaliar a ocorrência de agenesias dentais, classificando-se as ocorrências segundo sexo, raça e dente envolvido. Foram realizados exames clínicos e radiografias panorâmicas em 224 pacientes com idade média de 22 anos. A maior frequência de agenesia foi no gênero feminino. No grupo dos pré-molares, a mais alta incidência de agenesia foi de primeiros pré-molares superiores.

Com o objetivo de avaliar a prevalência de agenesias dentárias numa população, Grieco et al. realizaram um estudo, em 2007, nos pacientes em tratamento ortodôntico na Universidade da Cidade de São Paulo. O material examinado constituiu-se de 1.117 panorâmicas de pacientes acima de 9 anos de idade. A amostra foi estudada quanto à distribuição de prevalência entre os gêneros, os grupos raciais, os quadrantes dentários e entre os diversos grupos dentários. Foi verificado que a ocorrência de agenesia não esteve associada ao gênero, raça ou quadrantes. Com relação aos grupos de dentes, a maior prevalência foi verificada para os segundos pré-molares inferiores e incisivos laterais superiores.

Paula e Ferrer (2007) realizaram uma pesquisa em 800 radiografias panorâmicas, com o objetivo de avaliar a prevalência de hipodontia numa clínica de ortodontia na cidade de Goiânia. A faixa etária variou entre 12 e 53 anos de idade. Segundo os autores, na amostra estudada foram encontrados 759 dentes ausentes. A maior prevalência de agenesia foi de $3^{\circ}$ molar inferior, $360(1,4 \%)$, seguida por $3^{\circ}$ molar superior, $286(1,1 \%)$, incisivo lateral superior, $71(0,2 \%)$, pré-molar inferior, $26(0,1 \%)$, pré-molar superior, $14(0,05 \%)$, incisivo lateral inferior, $1(0,004 \%)$ e canino superior, $1(0,004 \%)$. Foram encontrados no sexo feminino 497 dentes ausentes, $(65,4 \%)$ em relação ao masculino, $262(34,5 \%)$.

A hipodontia de dentes permanentes foi avaliada por Goya et al. em 2008, a partir de radiografias panorâmicas de 2072 pacientes pediátricos na Universidade Nihon, em Matsudo, Japão. A prevalência encontrada foi de $8,7 \%$ em meninos e $10,8 \%$ nas meninas, e $9,4 \%$ para ambos os sexos combinados. A maioria dos casos $(67,8 \%)$ envolveu um ou dois dentes. 0 dente mais comumente ausente foi o segundo pré-molar inferior. Foi ainda observada uma elevada frequência de incisivos inferiores acometidos com a alteração (18,82\%), e, segundo os autores, esta parecia ser uma característica peculiar aos indivíduos de etnia asiática. A distribuição entre maxilar e mandíbula não teve associação significativa. 
Chung et al. (2008) estudaram a prevalência de hipodontia numa população coreana, bem como sua associação com o ausência congênita do terceiro molar. Os autores utilizaram 1622 radiografias panorâmicas divididas entre 611 homens e 1011 mulheres. A porcentagem de hipodontia encontrada foi de $11,2 \%$. Eles perceberam que era mais frequente na mandíbula do que na maxila, e os dentes mais comumente afetados foram os incisivos laterais (40\%), seguidos dos segundos pré-molares inferiores $(20,4 \%)$.

Borba et al. (2010), realizaram um estudo com o objetivo de avaliar a prevalência das agenesias dentais em pacientes com idades entre 7 e 16 anos, no município de Campo Grande, Mato Grosso do Sul, a partir da análise de radiografias panorâmicas obtidas no período de 2005 a 2007 . Os autores encontraram a presença de agenesia em $40,6 \%$ dos pacientes. Foi demonstrado não haver influência do gênero na ocorrência da agenesia dental. 0 dente que apresentou maior frequência deste tipo de anomalia foi o terceiro molar, seguido pelo segundo pré-molar (principalmente os inferiores), incisivo lateral superior e primeiro pré-molar superior. Embora não existindo diferença estatisticamente significativa em relação aos demais quadrantes, a localização mais frequente ocorreu na mandíbula.

Carvalho, Mesquita e Afonso (2011) realizaram um estudo com o objetivo de determinar a prevalência de anomalias de número na dentição de uma população portuguesa, através da análise de radiografias panorâmicas de pacientes que frequentaram a clínica da Faculdade de Medicina Dentária da Universidade do Porto (FMDUP). Obtiveram uma prevalência de agenesias dentárias, na dentição permanente, de $12,23 \%$, incluindo os $3 .{ }^{\circ}$ s molares, e de $6,47 \%$, se excluirmos estes dentes.

Malta (2012) estudou a prevalência das agenesias dentarias numa população de pacientes da Clínica Pedagógica de Medicina Dentaria da Faculdade Ciências da Saúde da Universidade Fernando Pessoa, na Paraíba. No estudo, foram selecionados 241 pacientes com idades compreendidas entre os 7 e os 18 anos de idade. 0 diagnóstico de agenesia dentária foi feito a partir da análise das radiografias panorâmicas contidas nos processos clínicos e com consulta das fichas clínicas, procedendo-se à coleta de dados sobre idade e gênero, excluindo os terceiros molares. Os dentes mais frequentemente ausentes foram os incisivos laterais superiores. A prevalência por gênero foi de $41,7 \%$ (masculino) e $58,3 \%$ (feminino); segundo as arcadas dentárias verificou-se $14,9 \%$ de agenesias no maxilar superior e $8,7 \%$ no inferior.

Trakinienė, Ryliškytė e Kiaušaitė (2013) realizaram um estudo na Universidade de Ciências da Saúde, da Lituânia, com a finalidade de determinar se o número de dentes com anomalias é mais freqüente em pacientes ortodônticos que na população comum. No estudo foram avaliados os registros de 824 pacientes ortodônticos (idade média 15,22 anos). A prevalência de hipodontia foi 17,11\%, sem diferença estatisticamente significante entre os gêneros. A ocorrência unilateral de agenesia dentária foi de 1,5 vezes mais comum do que a ocorrência bilateral. Os terceiros molares superiores e inferiores foram os dentes mais frequentemente afetados, seguidos pelo segundo pré-molar inferior.

Al Jawad et al. realizaram um estudo, no ano de 2015, com o objetivo de investigar a prevalência e distribuição de hipodontia nos dentes permanentes entre um grupo de pacientes do Hamad Medical, no Qatar. Os autores analisaram 1269 radiografias panorâmicas (674 de indivíduos do gênero feminino com idade média de $11,8 \pm 2,3$ e 595 do masculino, com idade média de 11,4 $\pm 2,2$ ). Todos os dentes permanentes foram investigados, exceto os terceiros molares. Relataram a prevalência de $6,2 \%$, sendo $8 \%$ entre as mulheres e $4,2 \%$ entre os homens. A maioria dos pacientes tinha um ou dois dentes faltando. Não houve diferenças significativas entre os lados direito e esquerdo para qualquer dente particular. Os dentes mais frequentemente em falta foram incisivos superiores laterais $(36,2 \%)$, seguidos pelo segundo pré-molar inferior (32,6\%) e segundo pré-molar superior $(20,2 \%)$. 
Gokkaya, Motro e Garkul (2015) realizaram um estudo retrospectivo, onde foram revisados os prontuários de pacientes ortodônticos turcos tratados entre 1994 e 2003. Um total de 1236 pacientes ortodônticos (507 meninas, 729 meninos) foi incluído no estudo. A idade das pacientes variou de 11 a 20 anos. Foram analisadas as diferenças na distribuição da hipodontia, sexo e tipo de má oclusão. Foram encontradas 82 crianças com hipodontia, das quais 45 meninas e 37 meninos. A prevalência foi de $7 \%$. 0 segundo pré-molar inferior foi o dente mais comumente ausente em 48 meninas e 26 meninos.

Hassan, Abuaffan e Hashim (2015) analisaram 1069 radiografias panorâmicas de pacientes sudaneses, com o objetivo de determinar a prevalência de hipodontia na dentição permanente. No estudo foram incluídos 760 mulheres e 309 homens, todos acima dos 8 anos de idade. A prevalência de hipodontia na amostra foi de $5,1 \%$, sendo mais frequente na mandíbula do que na maxila. Os dentes mais frequentemente afetados foram os incisivos laterais superiores, seguidos pelos segundos prémolares inferiores, segundo pré-molares superiores e incisivo lateral esquerdo mandibular.

Fekonja (2015) analisou a prevalência de hipodontia dos dentes permanentes, por meio de entrevistas e exames radiográficos orais e panorâmicas de 2546 eslovenos nascidos nos anos 1966, 1976, 1986 e 1996. Hipodontia foi observada em 175 indivíduos (6,9\%), excluindo os terceiros molares. Os dentes mais comumente acometidos foram o segundo pré-molar inferior $(38,8 \%)$, seguido do incisivo lateral superior $(32,6 \%)$. A maioria $(86,9 \%)$ dos pacientes com hipodontia apresentava falta de apenas um ou dois dentes.

\section{MÉTODOS E TÉCNICAS}

Cinco mil quinhentas e oitenta radiografias panorâmicas pertencentes ao arquivo da Disciplina de Patologia Bucal do Curso de Odontologia do UniFOA, Volta Redonda, Rio de Janeiro, obtidas no período de janeiro a dezembro de 2014 , foram avaliadas sobre a presença de imagens compatíveis com hipodontia.

Para avaliação foi utilizado negatoscópio com tela composta por diodos emissores de luz branca (LEDs) modelo Driller (VK Driller - Brasil), e com o auxílio de uma lupa com cabo, $60 \mathrm{~mm}$ de diâmetro, aumento de 2x, modelo LP-60 (Western - Brasil). As radiografias foram inicialmente avaliadas pelos alunos participantes da pesquisa, e posteriormente pelos professores orientadores.

Como critério de inclusão, foram consideradas todas as radiografias que apresentaram imagem de espaço compatível com ausência de germes dentários de dentes permanentes, e presença do dente decíduo correspondente retido.

Como critérios de exclusão, foram descartadas as radiografias que apresentaram imagem de espaço compatível com ausência de germes dentários de dentes permanentes e ausência concomitante do decíduo correspondente, devido a incerteza do diagnóstico, se induzida ou não; e os molares, por não haver como identificar apenas pelas radiografias se o germe não se desenvolveu ou o paciente foi submetido à exodontia dos mesmos.

Os dados foram catalogados em planilha utilizando-se o Microsoft Excel versão 2010 e os resultados foram apresentados sob a forma de tabelas.

Para avaliação dos critérios éticos, um protocolo foi encaminhado ao Comitê de Ética em Pesquisa com Seres Humanos do Centro Universitário de Volta Redonda, que emitiu parecer favorável à sua realização e com registro na Comissão Nacional de Ética em Pesquisa do Ministério da Saúde (CAAE - 25051213.6.0000.5237, Parecer $n^{\circ} 491510$ de 11/12/2013). 


\section{RESULTADOS}

Dentre as 5.580 radiografias examinadas, foram encontrados $77(1,38 \%)$ de indivíduos com imagem correspondente à ausência do germe dentário.

A idade variou de 6 a 54 anos, com média de 17,5 anos, e predominância na faixa etária entre 11 e 20 anos $(n=48)$ (Tabela 1).

Tabela 1 - Idade

\begin{tabular}{ccc}
\hline IDADE & $\mathbf{n}$ & $\%$ \\
\hline 6 a 10 & 11 & 14,28 \\
\hline 11 a 20 & 48 & 62,37 \\
\hline 21 a 30 & 13 & 16,88 \\
\hline 31 a 40 & 3 & 3,88 \\
\hline 41 a 50 & 0 & 0 \\
\hline 51 a 60 & 2 & 2,59 \\
\hline TOTAL & 77 & 100 \\
\hline
\end{tabular}

Fonte: dos autores.

Ocorreram 40 casos no gênero feminino $(51,95 \%)$ e 37 no masculino $(48,05 \%)$, numa relação 1,08X1 (Tabela 2).

Tabela 2 - Gênero

\begin{tabular}{ccc} 
& $\mathbf{n}$ & $\%$ \\
\hline Masculino & 37 & 48,05 \\
\hline Feminino & 40 & 51,95 \\
\hline TOTAL & 77 & 100 \\
\hline
\end{tabular}

Fonte: dos autores.

Em relação ao número de dentes com hipodontia por paciente, 53,24\% $(n=41)$ apresentaram 1 germe ausente, 36,36\% ( $n=28) 2$ germes, 3,90\% (n=3) 3 germes, 5,20\% (n=4) 4 germes, e $1,30 \%(n=1)$ 5 germes (Tabela 3). Quanto à distribuição pelo quantitativo de dentes com hipodontia em relação a gênero, foi observado que, de acordo com o aumento do número de dentes afetados, a relação Feminino $\mathrm{x}$ Masculino se inverteu (Tabela 4).

Tabela 3 - Número de dente com Hipodontia X Paciente

\begin{tabular}{ccc}
\hline Hipodontia & $\mathbf{n}$ & $\%$ \\
\hline 1 & 41 & 53,24 \\
\hline 2 & 28 & 36,36 \\
\hline 3 & 3 & 3,90 \\
\hline 4 & 4 & 5,20 \\
\hline 5 & 1 & 1,30 \\
\hline TOTAL & 77 & 100 \\
\hline
\end{tabular}

Fonte: dos autores. 
Tabela 4 - Número de dentes Hipodontia/Indivíduo X Gênero

\begin{tabular}{cccccc}
\hline Número de dentes com Hipodontia & Feminino $\mathbf{n}$ & $\%$ & Masculino $\mathbf{n}$ & $\%$ \\
\hline 1 & 24 & 31,17 & 17 & 22,07 \\
\hline 2 & 14 & 18,18 & 14 & 18,18 \\
\hline 3 & 0 & 0 & 3 & 3,90 \\
\hline 4 & 1 & 1,30 & 3 & 3,90 \\
\hline 5 & 1 & 1,30 & 0 & 0 \\
\hline TOTAL & $\mathbf{4 0}$ & $\mathbf{5 1 , 9 5}$ & $\mathbf{3 7}$ & $\mathbf{4 8 , 0 5}$ \\
\hline
\end{tabular}

Fonte: dos autores.

Quando observados os dentes/grupo de dentes afetados, considerando ainda que alguns indivíduos apresentaram mais de uma hipodontia, o $2^{\circ}$ pré-molar inferior foi o mais afetado, com $57,60 \%$ $(n=72)$, seguido do $2^{\circ}$ pré-molar superior, com $22,40 \%(n=28)$ (Tabela 5$)$.

Tabela 5 - Hipodontia por Grupo de Dentes

\begin{tabular}{lcc}
\hline \multicolumn{1}{c}{ Grupo de dentes } & $\mathbf{n}$ & $\%$ \\
\hline $2^{\circ}$ pré-molar superior & 28 & 22,40 \\
\hline $2^{\circ}$ pré-molar inferior & 72 & 57,60 \\
\hline $1^{\circ}$ pré-molar superior & 2 & 1,60 \\
\hline $1^{\circ}$ pré-molar inferior & 3 & 2,40 \\
\hline canino superior & 5 & 4,00 \\
\hline canino inferior & 0 & 0 \\
\hline incisivo lateral superior & 10 & 8,00 \\
\hline incisivo lateral inferior & 0 & 0 \\
\hline incisivo central superior & 0 & 0 \\
\hline incisivo central inferior & 5 & 4,00 \\
\hline TOTAL & $\mathbf{1 2 5}$ & $\mathbf{1 0 0}$ \\
\hline
\end{tabular}

Fonte: dos autores.

Ainda em relação aos dentes afetados, pode-se observar que a maioria dos casos de hipodontia foram encontrados na mandíbula $(64 \%$ e $n=80)$, estando numa relação de $1,77 \times 1$ em relação à maxila (36\% e $n=45)$ (Tabela 6).

Tabela 6 - Hipodontia por Grupo de Dentes/Arcada Dentária

\begin{tabular}{ccc}
\hline Grupo de dentes & $\mathbf{n}$ & $\%$ \\
\hline Maxilar & 45 & 36,0 \\
\hline Mandíbula & 80 & 64,0 \\
\hline TOTAL & 125 & 100 \\
\hline
\end{tabular}

Fonte: dos autores. 


\section{DISCUSSÃO}

Durante o atendimento odontológico, o Cirurgião Dentista se depara com ausências dentárias em indivíduos jovens, muitos deles sem história de exodontias pregressas e, em alguns casos, com a retenção do elemento decíduo correspondente. Frequentemente isso é confirmado com o auxílio de exames radiográficos, e, em outros casos, aparecendo como achado em pacientes submetidos a esse tipo de exame complementar.

O estudo das anomalias dentárias de desenvolvimento tem apresentado resultados nem sempre convergentes. Os índices de prevalência, distribuição por gênero, arcada e dentes envolvidos têm variado na literatura, segundo a população pesquisada.

Em nosso estudo, buscou-se avaliar a frequência de hipodontia, que consiste numa alteração significativa, uma vez que representa um importante fator etiológico da maloclusão dentária e de problemas estéticos.

Procurou-se ainda incluir, dentro da revisão da literatura realizada, estudos desenvolvidos em diferentes populações com diferentes etnias, para que se pudesse buscar comparações com a população brasileira.

A prevalência encontrada no presente estudo foi de 1,38\%, índice esse dentro da variação de $0,03 \%$ a 10,1\%, citada por Farias et al. (2006) e Scully (2009), e, recentemente, por Gokkaya, Motro e Garkul (2015). Foi observado porém, que o índice encontrado esteve abaixo dos estudos referenciados, estando mais próximo do trabalho realizado por Oliveira et al. (2001) em São Paulo (3,87\%), e mais distante dos estudos realizados por Borba et al. (2010) e Malta (2012), realizados nos estados do Mato Grosso do Sul e Paraíba, respectivamente, ambos acima dos $40 \%$. Após avaliar cuidadosamente a metodologia de cada autor, acreditamos inicialmente que essa grande diferença poderia ser atribuída à inclusão dos $3^{\circ} \mathrm{s}$ molares nos referidos estudos. Destacamos, porém, que outros estudos, como os de Girondi et al. (2006) em São Paulo, e Trakinienè, Ryliškytè e Kiaušaitè (2013) na Lituânia, incluíram os $3^{\circ}$ s molares, encontrando números bem inferiores (respectivamente 6,57\% e 17,11\%). Diante do relatado, não se pode atribuir qualquer fator que pudesse justificar a diferença relatada.

A idade variou de 6 a 54 anos, com média de 17,5 anos, e predominância na faixa etária entre 11 e 20 anos, faixa etária essa presente na maioria dos estudos aqui relatados, que nada mais representa do que a idade da maioria dos indivíduos que buscam tratamento estético/conservador, em especial o ortodôntico.

O gênero feminino foi o mais acometido, estando de acordo com os estudos de Castro et al. (2006), Paula e Ferrer (2007), Goya et al. (2008) Malta (2012) e Gokkaya, Motro e Garkul (2015). Nenhum estudo relatou maior acometimento do gênero masculino, sendo que nos estudos de Grieco et al. (2007) e Borba et al. (2010) foi citado que não houve diferenças estatísticas entre os gêneros. Em relação à distribuição pelo quantitativo de dentes com hipodontia, foi observado que, de acordo com o aumento do número de dentes afetados, a relação Feminino x Masculino se inverteu. Enquanto que nos pacientes com apenas 1 dente envolvido a relação foi de 1,41X1, nos pacientes com mais de um dente afetado a relação se modificou para $1 \times 1,25$, dado esse que não foi relatado em nenhum outro estudo, ficando apenas como registro para futuras comparações.

Em relação ao número de dentes com hipodontia por paciente, 53,24\% $(n=41)$ apresentaram 1 germe ausente e $36,36 \%$ (n=28) 2 germes, totalizando $89,6 \%$, estando de acordo com os números publicado por Goya et al. (2008), Fekonja (2015) e Al Jawad et al. (2015). 
Quando observados os dentes/grupo de dentes afetados, considerando ainda que alguns indivíduos apresentaram mais de uma hipodontia, o $2^{\circ}$ pré-molar inferior foi o mais afetado com $57,60 \%$ $(n=72)$, estando de acordo com os estudos de Grieco et al. (2007), Goya et al. (2008), Borba et al. (2010), Trakinienė, Ryliškytė e Kiaušaitė (2013), Fekonja (2015) e Gokkaya, Motro e Garkul (2015). Importante observar que apesar das diferentes etnias (Grieco et al. no estado de São Paulo; Goya et al. no Japão; Borba et al. no Estado do Mato Grosso do Sul; Trakinienė, Ryliškytė e Kiaušaitė na Lituânia; Fekonja na Eslovênia e Gokkaya, Motro e Garkul na Turquia), o $2^{\circ}$ pré-molar inferior manteve-se como o principal dente afetado. Como a principal faixa etária citada em nosso trabalho é semelhante às demais citadas, percebe-se que os dentes em desenvolvimento no período são os $2^{\circ}$ molares, os pré-molares e os caninos. Dentre esses, devido ao número nas arcadas (um total de 8 , contra 4 dos demais), os pré-molares são os mais passíveis de apresentar a alteração, o que poderia justificar sua relação como grupo de dentes mais afetado.

Ainda em relação aos dentes afetados, pode-se observar que a maioria dos casos de hipodontia foram encontrados na mandíbula $(64 \%$ e $n=80)$, estando numa relação de $1,77 \times 1$ em relação à maxila. Esse dado também foi observado nos estudos de Chung et al. (2008) e Hassan, Abuaffan e Hashim (2015). Malta (2012) observou maior prevalência na maxila, enquanto Goya et al. em (2008) relataram não haver diferenças entre as arcadas. Entendemos que a localização óssea maxila/mandíbula não foi fator determinante para a localização, estando possivelmente ligada ao acaso, haja vista o números de estudos em que esse dado não foi inserido.

\section{CONCLUSÃO}

Após a avaliação realizada, pode-se concluir que:

1. A prevalência de indivíduos com imagem correspondente à hipodontia foi de 1,38\%;

2. O gênero feminino foi ligeiramente mais acometido pela alteração, sendo o dente mais frequente o $2^{\circ}$ pré-molar inferior seguido do $2^{\circ}$ pré-molar superior.

Nossos resultados corroboram a importância do exame radiográfico detalhado e cuidadoso, o que contribui com um plano de tratamento eficaz, de acordo com as necessidades individuais do paciente. 


\section{REFERÊNCIAS}

AL JAWAD, F.H.A.; AL YAFEI, H.; AL SHEEB, M.; et al. Hypodontia prevalence and distribution pattern in a group of Qatari orthodontic and pediatric patients: A retrospective study. Europ J Dent v. 9, n. 2, p. 267-71, Apr-Jun 2015.

BRUNNER, V.; GUEDES-PINTO, A.C. Anomalias do desenvolvimento dental e sua importância na pratica clinica. Rev. APCD, v. 37, n. 1, p. 182-91, jan./fev. 1983.

BORBA, G.V.C; JUNIOR, J.C.B; PEREIRA, K.F.S; SILVA, P.G; Levantamento da prevalência de agenesias dentais em pacientes entre 7 e 16 anos. Rev Gaúcha de Odontologia, Porto Alegre, v. 58, n.1, p. 35-9, jan./mar. 2010.

CARVALHO, S.; MESQUITA, P.; AFONSO, A. Prevalência das anomalias de número numa população portuguesa. Estudo radiográfico. Rev Port Estomatol Med Dent Cir Maxilofac v. 52, n. 1, p. 7-12, 2011.

CASTRO, E.V.F.L.; CASTRO, A.L.; SALZEDAS, L.M.P.; JARDIM, P.T.C.; JARDIM, A.T.B. Agenesia e inclusão dental patológica. estudo clínico e radiográfico em pacientes. Rev. Fac. Odontol. Lins, Piracicaba v. 18, v. 1, p. 41-6, 2006.

CHUNG, C.J.; HAN, J.H.; KIM, K. H. The pattern and prevalence of hypodontia in Koreans. Oral Dis v. 14, p. 620-5, 2008.

FARIAS, L.A.G; SIMÕES, W; BOZZO, R.O; OLIVEIRA, P.A; Prevalência da Agenesia Dentária de Jovens do Gênero Feminino. RGO v. 54, n. 2, p. 115-8, abr./jun 2006.

FEKONJA, A. Hypodontia Prevalence over Four Decades in a Slovenian Population. J Esthetic Rest Dentist v. 27, n. 1, p. 37-43, 2015.

GARTNER, F.C; GOLDENBERG, C.F; A importância da radiografia panorâmica no diagnostico e no plano de tratamento ortodôntico na fase da dentadura mista. Rev. Portal Metodista, São Paulo, v. 17, n. 33, p. 102-8, jan./jun. 2009.

GIRONDI, J.R; PEREIRA, M.F; CAMPOS, P.S.F; PANELLA, J; Estudo da prevalência das anomalias dentárias de desenvolvimento em dada população, com o uso de radiografias panorâmicas, Rev Odonto da UNICID v. 18, n. 1, p. 15-21, Jan./Abr. 2006.

GÖKKAYA, B.; MOTRO, M.; KARGÜL, B. Prevalence and characteristics of non-syndromic hypodontia among Turkish orthodontic patient population. J Int Soc Prev Community Dent v. 5, n. 3, p. 170-5, 2015.

GOYA, H.A.; TANAKA, S.; MAEDA, T.; AKIMOTO, Y. An orthopantomographic study of hypodontia in permanent teeth of Japanese pediatric patients J Oral Science, v. 50, n. 2, p. 143-50, 2008.

GRIECO, F.A.D.; CARVALHO, P.E.G.; GUEDES-PINTO, GARI, D.G.; VALLE-CORROTTI, K.M. Prevalência de agenesia dentária em pacientes ortodônticos da cidade de São Paulo. RPG Rev Pós Grad v. 13, n. 4, p. 312-7, 2007.

HASSAN, D.A.; ABUAFFAN, A.H.; HASHIM, H.A. Prevalence of hypodontia in a sample of Sudanese orthodontic patients. J Orthodontic Sci v. 3, n. 3, p. 63-7, 2014. 
KAPÁDIA, H.; MUES, G.; D'SOUZA, R. Genes affecting tooth morfogenesis. Ortho Craniofac Res, v. 10, n. 4, p. 237-44, 2007.

KIGNEL, S. Estomatologia: Bases para do diagnóstico para o Clínico. $2^{\mathrm{a}}$ ed. São Paulo: Santos, 2013.

MALTA, A.M.A. Estudo de Prevalência das Agenesias Dentárias, numa população de pacientes da Clínica da Faculdade de Ciências da Saúde da Universidade Fernando Pessoa. (Dissertação). Programa de PósGraduação. Faculdade Ciências da Saúde, Universidade Fernando Pessoa. Porto, Portugal, 2012, 78p.

NANCI, A. Tem Cate Histologia Oral.8ª Ed. Rio de Janeiro: Elsevier, 2013.

NEVILLE, B.W.; DAMM, D.D.; ALLEN, C.M.; BOUQUOT, J.E. Patologia Oral \& Maxilofacial. $3^{\text {a }}$ ed. Rio de Janeiro: Elsevier, 2009.

OLIVEIRA, O.M.S.; PALLOS, D.; GIL, F.; CORTELLI, J.L. Prevalência de Hipodontia e alterações da anatomia dentária relacionadas Rev. Biociênc.Taubaté, v. 7, n. 2, p. 31-7, jul.-dez 2001.

PAATERO, Y.V. Stereoscopy in orthoradial pantomography of the jaws. Acta Radiologica v. 51, n. 6, p. 449-52, 1959.

PAULA, A.F.B; FERRER, K.J.N; Prevalência de agenesia em uma clínica ortodôntica de Goiânia. RGO v. 55, n. 2, p. 149-53, Porto Alegre, abr./jun. 2007.

REGEZI, J, A; SCIUBBA.J.J; JORDAN. R.C.K. Patologia Oral: correlações clinicopatológicas. $6^{\text {a }}$ ed: Rio de Janeiro: Elsevier, 2013.

ROBINSON, H.B.G.; MILLER, A.S. Color Atlas of Oral Pathology. 5ª ed. Philadelphia: Lippincot, 1990.

SAPP, P.; EVERSOLE, L. R.; WYSOCKI, G. P. Patologia bucomaxilofacial contemporânea. $2^{\mathrm{a}}$ ed. São Paulo: Santos, 2012.

SCULLY, C. Medicina oral e maxilofacial: bases do diagnóstico e tratamento. $2^{\mathrm{a}}$ ed. Rio de Janeiro: Elsevier, 2009.

TRAKINIENĖ, G.; RYLIŠKYTĖ, M.; KIAUŠAITĖ, A. Prevalence of teeth number anomalies in orthodontic patients. Stomatol, Baltic Dental and Maxillofac J. v. 15, p. 47-53, 2013. 